\title{
Quality Requirements for Genomic DNA Preparations and Storage Conditions for a High-Density Oligonucleotide Microarray
}

\author{
Kouichi Kurose,* Tomoko Koizumi, Jun Nishikawa, Keiko Maekawa, and Yoshiro Saito \\ Division of Medicinal Safety Science, National Institute of Health Sciences; 1-18-1 Kamiyoga, Setagaya-ku, Tokyo \\ 158-8501, Japan. Received June 13, 2012; accepted July 15, 2012
}

\begin{abstract}
High-density oligonucleotide microarrays are widely used in genome-wide association studies. The purpose of this study was to assess the influence of various factors during the preparation of DNA on genotype calling for the Affymetrix high-density oligonucleotide microarray 250K GeneChip. DNA was extracted from peripheral whole blood by solution-based and silica-membrane-based methods. Blood was stored at $4^{\circ} \mathrm{C}$ or $25^{\circ} \mathrm{C}$ for 4 or $24 \mathrm{~h}$, followed by DNA extraction. To examine the effects of freeze-thaw cycles, blood and DNA were also subjected to 5 and 10 or 20 of freeze-thaw cycles, respectively. The suitability of variously DNA preparations for the array was assessed by the call rate resulting from genotyping. All DNA samples showed mean call rates of more than 0.99 , which passed the quality criteria for genotyping (greater than 0.95). The results indicated that the solution-based method and the silica-membrane-based DNA extraction method could provide DNA of sufficient quality for genotyping. In addition, DNA quality suitable for high-density oligonucleotide microarrays is not strongly dependent on the preparation conditions under standard procedures.
\end{abstract} ment

Key words genome-wide association study; microarray; DNA preparation; storage condition; drug develop-

Recently, genome-wide association studies (GWASs) have been widely used to identify disease-susceptibility genes, pharmacogenomic genes, or genetic biomarkers. ${ }^{1-4)}$ Thousands of GWASs have been performed and the number of publications has increased exponentially year-on-year, ${ }^{5,6)}$ implying that findings from GWASs will lead to new therapeutic targets for drug development and novel diagnostic objectives for disease and drug responses. However, obtaining reliable GWAS results is the key to success.

Guidelines have been published from the International Conference on Harmonisation of Technical Requirements for Registration of Pharmaceuticals for Human Use concerning the use of genetic biomarkers derived from pharmacogenomic data in drug development and their proper usage. These guidelines comprise: 1) Definitions for genomic biomarkers, pharmacogenomics, pharmacogenetics, genomic data and sample categories (ICH E15) ${ }^{7}$; and 2) recommendations regarding context, structure, and format of regulatory submissions for qualification of genomic biomarkers (ICH E16). ${ }^{8)}$ However, requirements for DNA quality have neither been fully assessed nor issued as guidance in Japan. The U.S. Food and Drug Administration (FDA) and European Medicines Agency (EMA) issued draft guidance (clinical pharmacogenomics: premarketing evaluation in early phase clinical studies) and a reflection paper (pharmacogenomic samples, testing and data handling), respectively, both of which contain little description of recommended preparation/handling procedures for maintaining genomic DNA quality. ${ }^{9,10)}$ Practically, differences during the DNA preparation process, from blood collection to DNA storage, may affect genotyping results and their interpretation. Accordingly, we examined the influence of various factors, including methods of DNA extraction from whole blood, storage conditions of blood samples, and freeze-thaw cycles of DNA samples, on the genotype call rate, using a high-density oligonucleotide microarray that is widely used for GWAS.

The authors declare no conflict of interest.

\section{MATERIALS AND METHODS}

Blood Samples Human peripheral blood samples from five healthy donors were obtained from ProMedDx (Norton, MA, U.S.A.), in which ethylenediaminetetraacetic acid (EDTA) was used as an anticoagulant. The blood samples were frozen at $-80^{\circ} \mathrm{C}$ within $2 \mathrm{~h}$ after blood collection and kept at $-80^{\circ} \mathrm{C}$ until use. The ethics committees of the $\mathrm{Na}-$ tional Institute of Health Sciences approved this study. Written informed consent was obtained from all donors.

Extraction of Genomic DNA The whole blood was stored at 4 or $25^{\circ} \mathrm{C}$ for 0,4 or $24 \mathrm{~h}$ before extraction. Genomic DNA was extracted from the peripheral whole blood by two different methods: a solution-based method using a Wizard ${ }^{\circledR}$ Genomic DNA Purification Kit (Promega KK, Tokyo, Japan) and a silica-membrane-based method using NucleoSpin ${ }^{\circledR}$ Tissue (Macherey-Nagel, Düren, Germany). Both methods were performed according to the manufacturer's instructions. Briefly, the solution-based method (method 1) was based on a 4-step process. The first step involved lysis of the red blood cells, followed by lysis of the white blood cells and their nuclei. The cellular proteins were then removed by a salt-precipitation step, which left the high molecular weight genomic DNA in solution. Finally, the genomic DNA was concentrated and desalted by isopropanol precipitation, and then dissolved in TE buffer (10mm Tris-Cl, $\mathrm{pH} 7.4,1 \mathrm{~mm}$ EDTA, $\mathrm{pH}$ 8.0). The silica-membrane-based method (method 2) was simpler and quicker than method 1. Lysis was achieved by incubation of the blood sample in a proteinase K/SDS solution. Binding of DNA to the silica-membrane in the NucleoSpin ${ }^{\circledR}$ Tissue columns was effected by the addition of chaotropic salts and ethanol to the lysate. Contaminants were removed by subsequent washing with two different buffers. Pure genomic DNA was finally eluted using a low ionic strength buffer $(5 \mathrm{~mm}$ Tris-Cl, $\mathrm{pH}$ 8.5). Method 1 took more than $3.5 \mathrm{~h}$, whereas method 2 took only $30 \mathrm{~min}$.

Exposure of Blood and DNA Samples to Freeze-Thaw 
Cycles To examine the effect of freeze-thaw, blood and DNA samples were exposed to freeze-thaw cycles by placing the set of samples into a $-80^{\circ} \mathrm{C}$ freezer for $10 \mathrm{~min}$ and then moving them into a water bath at $25^{\circ} \mathrm{C}$ until they were completely thawed. The freeze-thaw numbers were set at 5 for blood, and 10 or 20 for DNA, which are more excessive numbers than those usually accepted in regular laboratory work.

Assessment of DNA Quality and Genotyping The quality of the DNA prepared under various conditions was assessed by the genotyping call rate. Genotyping was performed, using $250 \mathrm{ng}$ of DNA as starting material, with an Affymetrix GeneChip Human Mapping 250K Nsp Array (Affymetrix Inc., Santa Clara, CA, U.S.A.) containing 262264 SNPs, according to the standard protocol recommended by the manufacturer. Fluorescence intensities were quantified using the Affymetrix array scanner 3000 7G. Data management and analyses were performed using the Affymetrix GeneChip Operating Software 1.4 client. Genotyping calls were determined from fluorescent intensities using the GeneChip Genotyping Analysis Software 4.1 and the Bayesian robust linear model with Mahalanobis distance classifier (BRLMM) algorithm. ${ }^{11)}$ A call rate of more than $95 \%$ was defined as qualifying for GWAS.

Statistical Analyses Comparisons of genotyping call rates were analyzed by one-way analysis of variance (ANOVA), with repeated measurements to evaluate the influence of the various preparation conditions on DNA quality. A $p$-value of less than 0.05 was considered statistically significant.

\section{RESULTS AND DISCUSSION}

The examined storage conditions for blood samples, methods for DNA extraction, and freeze-thaw cycles for blood and DNA are shown in Table 1 . DNA was genotyped by a $250 \mathrm{~K}$ SNP microarray to assess the quality requirements of various DNA preparations for GWAS. All conditions for DNA preparations/storage passed the quality criteria for genotyping, giving a call rate greater than 0.95 (Fig. 1). ANOVA showed that there were no statistically significant differences in the call rates among the nine different preparations of DNA $(F(8,32)=$ 1.4896, $p=0.1999$ ).

Influence of the DNA Extraction Methods on Genotype Calling Immediately after the frozen blood of five individuals was thawed, an aliquot of each DNA was extracted by the two different methods. The mean genotype call rate from method 1 was 0.9961 , and from method 2 was 0.9953 (Fig. 1 , columns 1,8$)$, both of which exceed the quality criteria,

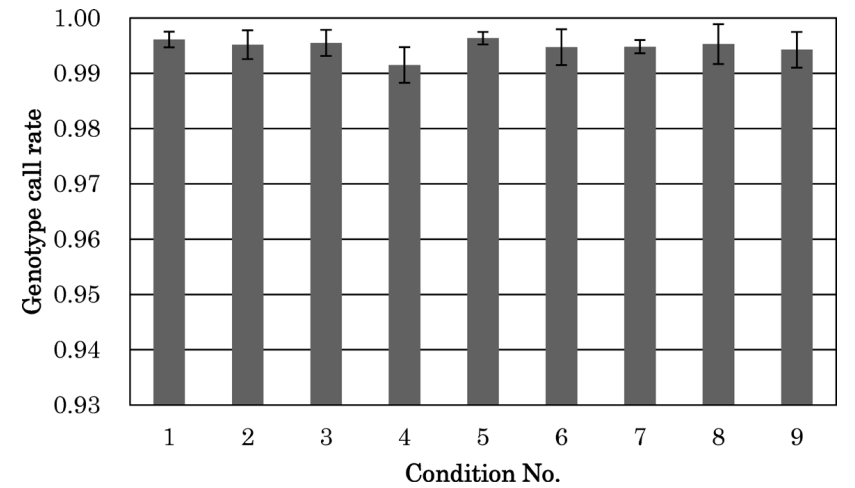

Fig. 1. Mean Genotype Call Rates for Affymetrix High-Density Oligonucleotide Microarrays by Genomic DNAs from Nine Different Preparative Conditions

The data represent the mean \pm S.D. of five individuals. The numbers under each column indicate the condition No. referred to in Table 1. There were no statistically significant differences among the call rates, as determined by one-way ANOVA with repeated measurements $(F(8,32)=1.4896, p=0.1999)$.

indicating that the quality of both methods was sufficient for high-density oligonucleotide microarrays. Method 1, however, is more complicated than method 2, and takes much longer to complete.

Influence of Blood Storage Conditions on Genotype Calling The effects of blood storage temperature and storage duration on genotype calling were assessed. Immediately after the frozen blood from five individuals was thawed, an aliquot of each sample was stored at 4 or $25^{\circ} \mathrm{C}$ for 4 or $24 \mathrm{~h}$ (Table 1). DNA was then extracted by method 1 and subjected to genotyping. All the mean call rates indicated high quality samples, with more than $99 \%$ ranging from 0.9915 to 0.9963 (Fig. 1, columns 4-7). The effect of repeated cycles of freezing and thawing during blood storage was then evaluated for five cycles. After the freeze-thaw cycles (between $-80^{\circ} \mathrm{C}$ and $25^{\circ} \mathrm{C}$ ) were completed, DNA was immediately extracted by method 2 and subjected to genotyping. The mean genotype call rate was 0.9942 (Fig. 1, column 9), which was of a similar high quality as that without freeze-thaw (Fig. 1, column 8). These results showed that none of the conditions for blood storage tested in this study had a negative effect on the genotype call rate. Therefore, it would be acceptable for preparing DNA for a GWAS study, even if the collected blood was accidentally left at room temperature for $24 \mathrm{~h}$ or was subjected up to five freeze-thaw cycles, for example by repeated power failures.

Influence of Freeze-Thaw Cycles of DNA on Genotype

Table 1. Blood Storage Conditions, DNA Extraction Methods, and Freeze-Thaw Cycles

\begin{tabular}{|c|c|c|c|c|}
\hline Condition No. & Blood storage & DNA extraction & Freeze-thaw cycles (blood) & Freeze-thaw cycles (DNA) \\
\hline 1 & $0 \mathrm{~h}$ & Solution-based & - & - \\
\hline 2 & $0 \mathrm{~h}$ & Solution-based & - & 10 \\
\hline 3 & $0 \mathrm{~h}$ & Solution-based & - & 20 \\
\hline 4 & $4^{\circ} \mathrm{C}, 4 \mathrm{~h}$ & Solution-based & - & - \\
\hline 5 & $25^{\circ} \mathrm{C}, 4 \mathrm{~h}$ & Solution-based & - & - \\
\hline 6 & $4^{\circ} \mathrm{C}, 24 \mathrm{~h}$ & Solution-based & - & - \\
\hline 7 & $25^{\circ} \mathrm{C}, 24 \mathrm{~h}$ & Solution-based & - & - \\
\hline 8 & $0 \mathrm{~h}$ & Silica-membrane & - & - \\
\hline 9 & $0 \mathrm{~h}$ & Silica-membrane & 5 & - \\
\hline
\end{tabular}


Calling The effect of further repeated cycles of freezing and thawing during the DNA storage was evaluated. The freezethaw cycles (between $-80^{\circ} \mathrm{C}$ and $25^{\circ} \mathrm{C}$ ) were conducted 10 and 20 times for samples prepared by method 1 . After the cycles were complete, the DNA samples were subjected to genotyping using the microarray. The mean call rates were 0.9952 and 0.9955 for 10 and 20 cycles, respectively (Fig. 1, columns $2,3)$, which were of a similar high quality as that without freeze-thaw (Fig. 1, column 1). In general, DNA solutions are stored frozen until analyzed, and thawed for subsequent analysis as necessary. The freeze-thaw process is repeated until analyses are completed, which could involve degradation of the DNA. However, our findings indicated that the freezing and thawing process (up to 20 cycles) inflicted no significant damage on the DNA in terms of the genotyping call rate.

\section{CONCLUSION}

In this study, we examined various possible factors that could affect genomic DNA quality, including the DNA extraction method and storage conditions of blood and DNA, by assessing genotype call rate for high-density oligonucleotide microarrays. There were no statistically significant differences in the call rates among the different preparations of DNA examined. All the methods and conditions tested in this study resulted in high quality DNA that met the standard for GWAS, as determined by the genotype call rate. Up to $24 \mathrm{~h}$ at $25^{\circ} \mathrm{C}$ storage of blood, five freeze-thaw cycles of whole blood, and at least 20 freeze-thaw cycles of DNA were acceptable. The easy and fast silica-membrane-based DNA extraction method generated DNA of similar quality to the solution-based method. As for long-term storage of DNA, genotype call rates of more than $99 \%$ were reported by other researchers using DNA samples stored at $4^{\circ} \mathrm{C}$ for more than 10 years in Tris-EDTA buffer. ${ }^{12}$ Therefore, it is expected that DNA of suitable quality for high-density oligonucleotide microarrays could be obtained if treated using conventional manipulations and under standard conditions.

Acknowledgments This study was supported in part by Health and Labour Sciences Research Grants from the Ministry of Health, Labour and Welfare, and by the Japan Health Sciences Foundation (Public-private sector joint research on Publicly Essential Drugs).

\section{REFERENCES}

1) SEARCH Collaborative Group, Link E, Parish S, Armitage J, Bowman L, Heath S, Matsuda F, Gut I, Lathrop M, Collins R. SLCO1B1 variants and statin-induced myopathy-a genome-wide study. $N$. Engl. J. Med., 359, 789-799 (2008).

2) Collins FS. Reengineering translational science: the time is right. Sci. Transl. Med., 3, 90cm17 (2011).

3) Tohkin M, Kaniwa N, Saito Y, Sugiyama E, Kurose K, Nishikawa J, Hasegawa R, Aihara M, Matsunaga K, Abe M, Furuya H, Takahashi Y, Ikeda H, Muramatsu M, Ueta M, Sotozono C, Kinoshita $\mathrm{S}$, Ikezawa Z. A whole-genome association study of major determinants for allopurinol-related Stevens-Johnson syndrome and toxic epidermal necrolysis in Japanese patients. Pharmacogenomics J., in press.

4) Kurose K, Hiratsuka K, Ishiwata K, Nishikawa J, Nonen S, Azuma J, Kato M, Wakeno M, Okugawa G, Kinoshita T, Kurosawa T, Hasegawa R, Saito Y. Genome-wide association study of SSRI/ SNRI-induced sexual dysfunction in a Japanese cohort with major depression. Psychiatry Res., in press.

5) Begum F, Ghosh D, Tseng GC, Feingold E. Comprehensive literature review and statistical considerations for GWAS meta-analysis. Nucleic Acids Res., 40, 3777-3784 (2012).

6) Hindorff LA, MacArthur J, Junkins HA, Hall PN, Klemm AK, Manolio TA. "A Catalog of Published Genome-Wide Association Studies.”〈http://www.genome.gov/gwastudies〉, cited 4 June 2012.

7) ICH E15. "Definitions for Genomic Biomarkers, Pharmacogenomics, Pharmacogenetics, Genomic Data and Sample Coding Categories."〈http://www.ich.org/fileadmin/Public Web Site/ICH Products/Guidelines/Efficacy/E15/Step4/E15_Guideline.pdf), cited 4 June 2012.

8) ICH E16. "Biomarkers Related to Drug Orbiotechnology Product Development: Context, Structure and Format of Qualification Submissions": 〈http://www.ich.org/fileadmin/Public_Web_Site/ICH Products/Guidelines/Efficacy/E16/Step4/E16_Step_4.pdf), cited 4 June 2012.

9) "Guidance for Industry. Clinical Pharmacogenomics: Premarketing Evaluation in Early Phase Clinical Studies. Draft Guidance.” http:// www.fda.gov/downloads/Drugs/GuidanceComplianceRegulatoryInformation/Guidances/UCM243702.pdf), cited 4 June 2012.

10) "Reflection Paper on Pharmacogenomic Samples, Testing and Data Handling.” 〈http://www.ema.europa.eu/docs/en_GB/document_library/Scientific_guideline/2009/09/WC500003864.pdf), cited 4 June 2012.

11) Rabbee N, Speed TP. A genotype calling algorithm for affymetrix SNP arrays. Bioinformatics, 22, 7-12 (2006).

12) Xing J, Watkins WS, Zhang Y, Witherspoon DJ, Jorde LB. High fidelity of whole-genome amplified DNA on high-density single nucleotide polymorphism arrays. Genomics, 92, 452-456 (2008). 